\title{
Asymmetric PPCys: Strongly fluorescing NIR labels $\dagger$
}

\author{
Georg Michael Fischer, Christian Jüngst, Magnus Isomäki-Krondahl, Dominik Gauss, \\ Heiko Michael Möller, Ewald Daltrozzo and Andreas Zumbusch*
}

Received 12th March 2010, Accepted 24th May 2010

First published as an Advance Article on the web 11th June 2010

DOI: $10.1039 / \mathbf{c 0 c 0 0 3 5 9 j}$

By a stepwise synthesis strategy biofunctionalized Pyrrolopyrrole Cyanines (PPCy) with an asymmetric substitution pattern were obtained. These exhibit extremely strong and narrowband NIR absorption and fluorescence. Internalization of a peptide bound PPCy is demonstrated using live cell microscopy.

Fluorescence microscopy is one of the key technologies in modern biology. ${ }^{1}$ It relies on the availability of strongly fluorescing and highly photostable chromophores, which can be functionalized for binding to biological target structures. ${ }^{2}$ The desire to investigate more complex biological samples, especially thick tissues, however, necessitates new methodological developments. One is the development of new fluorophores absorbing and emitting in the near infrared (NIR) spectral region. ${ }^{3}$ NIR excitation and detection offers many important advantages: (i) great sample penetration depths due to weak absorption and strongly reduced scattering; (ii) sensitive detection because of negligible NIR autofluorescence background; and (iii) less photodamage compared to visible excitation. ${ }^{4}$ These are reasons which motivated the recent development of a broad variety of new NIR-fluorophores. ${ }^{5}$ An important aspect here is the increase of the typically very low fluorescence quantum yields in the NIR.

In earlier work, we have described the synthesis and spectroscopic characterisation of pyrrolopyrrole cyanine dyes (PPCy), a new class of NIR dyes and fluorophores. ${ }^{6}$ They are obtained by heating a diketopyrrolopyrrole (DPP 1) and a heteroaromatic acetonitrile (HAA 3) in the presence of an excess of phosphoroxychloride. The resulting condensation products feature strong and narrow band absorption in the NIR. Solutions of these compounds do not yet fluoresce. Extraordinarily strong fluorescence at NIR wavelengths can however be induced by stiffening the PPCys through complexation with either $\mathrm{BF}_{2}$ or $\mathrm{BPh}_{2}$. Interestingly and in contrast to many other NIR dyes, PPCys show hardly any absorption in the visible spectral range which makes them attractive candidates for applications necessitating selective NIR absorption. By variation of the heterocyclic endgroups and the complexing agent, the absorption maximum can be shifted over a broad spectral range.

Fachbereich Chemie and Center of Applied Photonics, Universität Konstanz, 78457 Konstanz, Germany.

E-mail: andreas.zumbusch@uni-konstanz.de; Fax: + 49 7531-883870; Tel: +49 7531-2357

$\dagger$ Electronic supplementary information (ESI) available: Synthesis and characterisation of the intermediates and the final compounds. Absorption spectra of the dyes and details of the live cell imaging. See DOI: $10.1039 / \mathrm{c} 0 \mathrm{cc} 00359 \mathrm{j}$
PPCys show high chemical stability and photostability in nonpolar solvents such as chloroform or toluene. In the PPCys reported so far, the alkyl chains which are necessary for their synthesis complicate their use in polar or aqueous environment. Nevertheless, first in vivo experiments have been carried out by presolving the dyes in unpolar solvents and subsequently diluting them in buffer. This allowed fluorescence and fluorescence lifetime imaging in live mice, where PPCys exhibited good stability, bright fluorescence, very long fluorescence lifetimes, and no adverse effects in mice. ${ }^{7}$ While these results are promising, labeling applications with a more general scope require the functionalization and selective binding of PPCys to biological target molecules. Here, we report the implementation of a synthetic strategy improving the solubility in polar solvents and leading to functionalized PPCys. The resulting chromophores feature very strong absorptions above $730 \mathrm{~nm}$ and extraordinarily high fluorescence quantum yields in the NIR region.

The scheme for the synthesis of PPCys as it has been followed earlier ( $c f$. Scheme 1 in ref. $6 a$ ) leads to symmetric 1 : 2 condensation products. The high symmetry of these would lead to bifunctionalization, which is undesirable for labeling applications. Monofunctionalization requires a different strategy. Our rationale to reach this aim is to assemble the chromophores in a stepwise manner. For this purpose, the monosubstituted $1: 1$ condensation products of a DPP (1) with a HAA (3) have to be isolated (Scheme 1). Previously, this has only been achieved for the PPCy resulting as the $4-(N$-methyl- $N$-octyl amino)phenyl DPP $1: 1$ condensation product. ${ }^{6}$ All other PPCys were obtained as $1: 2$ condensation products. Mono-phosphorylated intermediates (2) from the reaction of a DPP with $\mathrm{POCl}_{3}$ have however been reported. ${ }^{8}$ These can be isolated in good yields by removing excess $\mathrm{POCl}_{3}$ in vacuum. The intermediates thus obtained can be dissolved in abs. THF and reacted with one equivalent HAA (3) to the monosubstituted product (4). The longest wavelength absorption $\left(\mathrm{S}_{0} \rightarrow \mathrm{S}_{1}\right)$ of $\mathbf{4}$ is broad with rather intense vibronic bands. As is the case for symmetrically substituted PPCys, solutions of $\mathbf{4}$ do not show fluorescence at room temperature. Complexation with $\mathrm{BF}_{2}$ leads to products (5) with $\mathrm{S}_{0} \rightarrow \mathrm{S}_{1}$ transitions whose intensity ratio of the purely electronic 00 -transition $v s$. the vibronic $01,02, \ldots$ transitions, as reflected by the Franck-Condon factors, are shifted in favour of the 00-transition. The $\mathrm{BF}_{2}$-complexes 5 exhibit strong fluorescence with Stokes shifts well above $1100 \mathrm{~cm}^{-1}$ ( $c f$. Table 1 and ESI $\dagger$ ).

The monosubstituted PPCys (4) serve as the starting material for the synthesis of asymmetrically bisubstituted PPCys. For this purpose, $\mathbf{4}$ is reacted with a second equivalent of a HAA (3) leading to asymmetric H-PPCys (6) in yields 


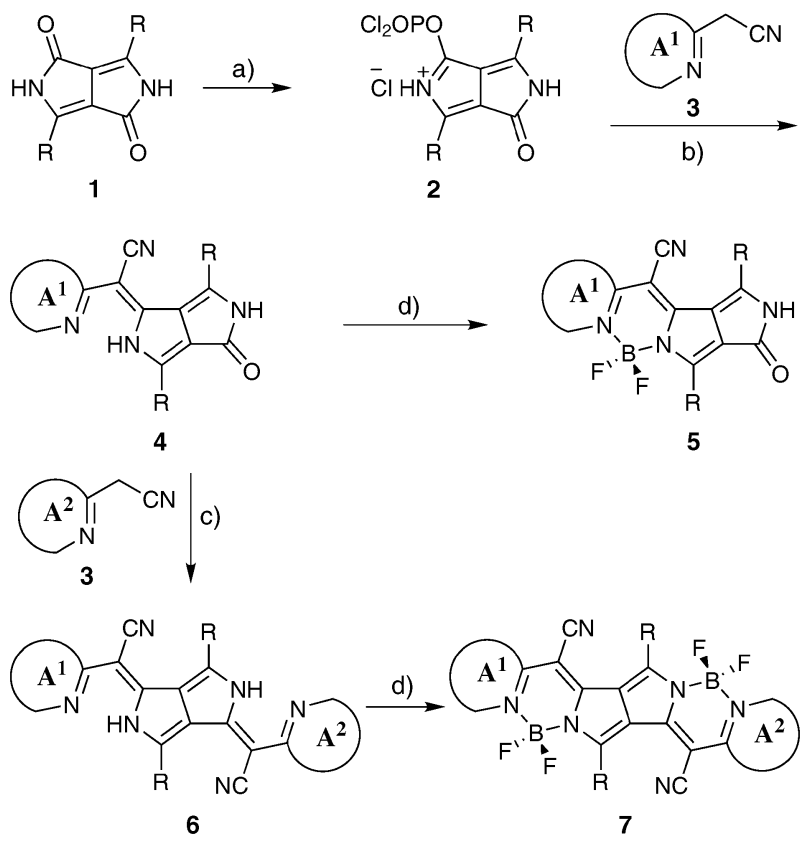

Scheme 1 Reagents and conditions: (a) $\mathrm{POCl}_{3}$, reflux; (b) absolute THF, reflux; (c) absolute toluene, $\mathrm{POCl}_{3}$, reflux; and (d) methylene chloride, di-iso-propylethylamine, $\mathrm{BF}_{3} \cdot \mathrm{OEt}_{2}$, reflux; $\mathrm{R}=p$-octyloxyphenyl; 3: (4,6-dimethyl-pyrimidine-2-yl)-acetonitrile (3a), (5-tertbutyl-benzooxazol-2-yl)-acetonitrile (3b), (6-methyl-pyridin-2-yl)-acetonitrile (3c), 2-(6-tert-butylbenzothiazol-2-yl)-acetonitrile (3d), 2-(6-tert-butylquinoline-2-yl)-acetonitrile (3e), 2-(6-bromoquinoline-2-yl)-acetonitrile (3f), quinoxalin-2-yl-acetonitrile (3g), tert-butyl 5-(2-(cyanomethyl)quinolin6-yl)pent-4-ynoate (3h). A: aromatic ring.

Table 1 Yield and spectroscopic data of the first electronic transition $\left(\mathrm{S}_{0} \leftrightarrow \mathrm{S}_{1}\right)$ of $\mathbf{4}$ and their $\mathrm{BF}_{2}$-complexes $\mathbf{5}^{a}$

\begin{tabular}{lllllll}
\hline & Yield & $\lambda_{00} \mathrm{~A} / \mathrm{nm}$ & $\varepsilon_{00} / \mathrm{M}^{-1} \mathrm{~cm}^{-1}$ & $\lambda_{00}{ }^{\mathrm{F}} / \mathrm{nm}$ & $\Delta \tilde{\nu}_{\mathrm{A}-\mathrm{F}} / \mathrm{cm}^{-1}$ & $\Phi_{\mathrm{F}}$ \\
\hline 4d & 40 & 618 & 33000 & - & - & - \\
4e & 84 & 611 & 37000 & - & - & - \\
5d & 44 & 618 & 43000 & 672 & 1320 & 0.50 \\
5e & 72 & 630 & 58000 & 678 & 1130 & 0.40
\end{tabular}

${ }^{a}$ In chloroform at room temperature, absorption/emission wavelength $\lambda_{00} \mathrm{~A} / \lambda_{00} \mathrm{~F}$, Stokes shift $\Delta \tilde{\nu}_{\mathrm{A}-\mathrm{F}}$, molar decadic absorption coefficient $\varepsilon_{00}$, fluorescence quantum yield $\Phi_{\mathrm{F}}$.

between 58 and $87 \%$. Complexation with $\mathrm{BF}_{3} \cdot \mathrm{OEt}_{2}$ yields asymmetric $\mathrm{BF}_{2}$-PPCys (7) between 60 and 86\% (Scheme 1). Obviously, as in the case of the classical cyanine cations ${ }^{9 a}$ and both the cationic and anionic cyanines, ${ }^{9 b}$ the asymmetric structure, with two different terminal groups $\mathrm{A}^{1}$ and $\mathrm{A}^{2}$, does not produce a significant deviation from the $C_{2}$ symmetry of

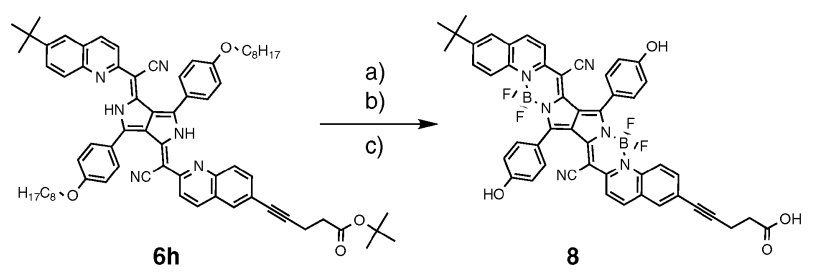

Scheme 2 Reagents and conditions: (a) TFA, $\mathrm{CH}_{2} \mathrm{Cl}_{2}$, reflux; (b) $\mathrm{CH}_{2} \mathrm{Cl}_{2}$, DIPEA, $\mathrm{BF}_{3} \cdot \mathrm{OEt}_{2}$, reflux; and (c) $\mathrm{CH}_{2} \mathrm{Cl}_{2}, \mathrm{BBr}_{3}$, room temperature.

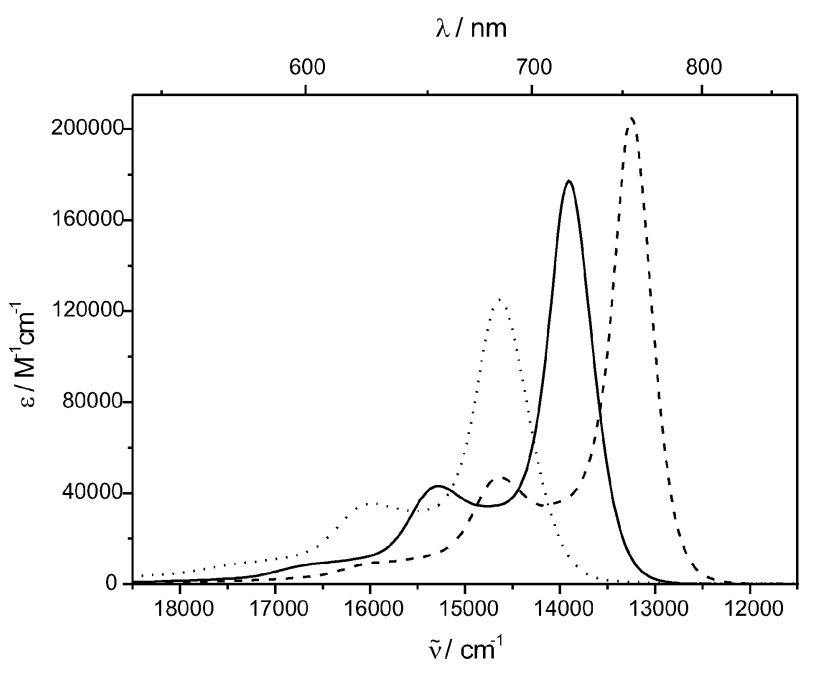

Fig. 1 Absorption of 7a: $\mathrm{A}^{1}=4,6$-dimethyl-pyrimidine (3a) $\mathrm{A}^{2}=6$-tert-butylquinoline (3e) (solid line) and the corresponding symmetric PPCys ${ }^{6}: \mathrm{A}^{1}=\mathrm{A}^{2}=\mathbf{3} \mathbf{a}\left(\right.$ dotted line) and $\mathbf{7 e}: \mathrm{A}^{1}=\mathrm{A}^{2}=\mathbf{3 e}$ (dashed line).

the electron density distribution, which is relevant for the Franck-Condon factors of the first electronic transition. Thus the spectral properties (absorption maxima, extinction coefficients and oscillator strengths) of the $\mathrm{A}^{1} \mathrm{~A}^{2}$ types lie exactly between the corresponding values of the symmetrical $\mathrm{A}^{1} \mathrm{~A}^{1}$ and the $\mathrm{A}^{2} \mathrm{~A}^{2}$ types (see Fig. 1) supporting the classification of PPCys as typical cyanine dyes. The heteroaromatic substituents however shift the energy of the observed electronic transitions in a fashion which is also known from other cyanine chromophores. 9 Table 2 shows that by suitable combinations of $\mathbf{3}$, the absorption maxima can be shifted at will in a broad spectral range.

While the one-pot synthesis described earlier leads to symmetric PPCys $\left(\mathrm{A}^{1}=\mathrm{A}^{2}\right)$, the stepwise synthesis of the chromophores now permits the introduction of a single functional group. As an example, we introduced a carboxylic acid function by a Sonogashira reaction of $\mathbf{3 f}$ with tert-butyl-4pentyonate which yields $\mathbf{3 h}$. $\mathbf{3 h}$ can be converted with $\mathbf{4 e}$ to the asymmetric H-PPCy $\mathbf{6 h}$, which is deprotected to the free acid $\mathbf{6 i}$, and subsequently complexed to $\mathbf{7 i}$. All these dyes bear unfavorable octyl chains which are helpful in increasing the solubility during the syntheses and purifications, but hamper their use in polar solvents and especially in water. They can be removed by cleaving the octyl ethers with $\mathrm{BBr}_{3}$ which leads to derivative 8 (Scheme 2) which is insoluble in unpolar solvents such as methylene chloride, but sufficiently soluble for coupling reactions in polar solvents like NMP. This allows its coupling to biological target molecules, e.g. peptides. ${ }^{10}$

As a demonstration for the suitability of this approach for applications of asymmetric PPCys as labels in live cell fluorescence imaging, we linked $\mathbf{8}$ to the $\mathrm{N}$-terminus of an $\mathrm{Arg}_{9}$ peptide that is well known as a cell penetrating peptide. ${ }^{11}$ This construct was then used to incubate HeLa cells which were grown in microscope dishes for $30 \mathrm{~min}$ ( $c f$. ESI $\dagger) .{ }^{12}$ After washing, live cell images were taken with a standard confocal microscope. It can clearly be seen that the $\mathbf{A r g}_{9} \mathbf{- 8}$ constructs were internalized into the cells in the form of small aggregates 
Table 2 Spectroscopic data of the first electronic transition $\left(\mathrm{S}_{0} \leftrightarrow \mathrm{S}_{1}\right)$ of the H-PPCys 6 and the BF $\mathrm{BPCys}_{2}$ 7: $\mathrm{A}^{1}=\mathbf{3} \mathrm{e}^{a}$

\begin{tabular}{|c|c|c|c|c|c|c|c|c|c|c|c|}
\hline $\mathrm{A}^{2}$ & 6 & $\lambda_{00}^{\mathrm{A}} / \mathrm{nm}$ & $\varepsilon_{00} / \mathrm{M}^{-1} \mathrm{~cm}^{-1}$ & $f$ & 7 & $\lambda_{00}^{\mathrm{A}} / \mathrm{nm}$ & $\varepsilon_{00} / \mathrm{M}^{-1} \mathrm{~cm}^{-1}$ & $f$ & $\lambda_{00}^{\mathrm{F}} / \mathrm{nm}$ & $\Delta \tilde{\nu}_{\mathrm{A}-\mathrm{F}} / \mathrm{cm}^{-1}$ & $\Phi_{\mathrm{F}}$ \\
\hline $3 \mathbf{a}$ & $6 a$ & 708 & 100000 & 0.62 & $7 a$ & 719 & 178000 & 0.76 & 740 & 400 & 0.66 \\
\hline 3b & $\mathbf{6 b}$ & 720 & 108000 & 0.69 & $7 b$ & 718 & 162000 & 0.77 & 739 & 350 & 0.58 \\
\hline $3 c$ & 6c & 718 & 97000 & 0.61 & $7 c$ & 730 & 179000 & 0.76 & 751 & 350 & 0.58 \\
\hline 3d & 6d & 734 & 116000 & 0.72 & $7 d$ & 742 & 191000 & 0.79 & 759 & 350 & 0.60 \\
\hline $3 \mathbf{e}$ & $6 e$ & 731 & 118000 & 0.71 & $7 e$ & 754 & 205000 & 0.83 & 773 & 300 & 0.59 \\
\hline $3 f$ & $6 f$ & 734 & 122000 & 0.70 & $7 f$ & 758 & 236000 & 0.86 & 774 & 300 & 0.59 \\
\hline 3g & $6 \mathrm{~g}$ & 739 & 132000 & 0.73 & $7 \mathrm{~g}$ & 770 & 213000 & 0.83 & 786 & 300 & 0.41 \\
\hline \multirow[t]{2}{*}{$3 \mathbf{h}$} & $6 \mathrm{~h}$ & 739 & 134000 & 0.76 & - & - & - & - & - & 一 & 一 \\
\hline & $6 i$ & 739 & 134000 & 0.76 & $7 \mathbf{i}$ & 763 & 244000 & 0.91 & 782 & 300 & 0.56 \\
\hline
\end{tabular}

${ }^{a}$ In chloroform at room temperature, absorption/emission wavelength $\lambda_{00}{ }^{\mathrm{A}} / \lambda_{00}{ }^{\mathrm{F}}$, molar decadic absorption coefficient $\varepsilon_{00}$, oscillator strength $f$, Stokes shift $\Delta \tilde{\nu}_{\mathrm{A}-\mathrm{F}}$ and fluorescence quantum yield $\Phi_{\mathrm{F}}$.

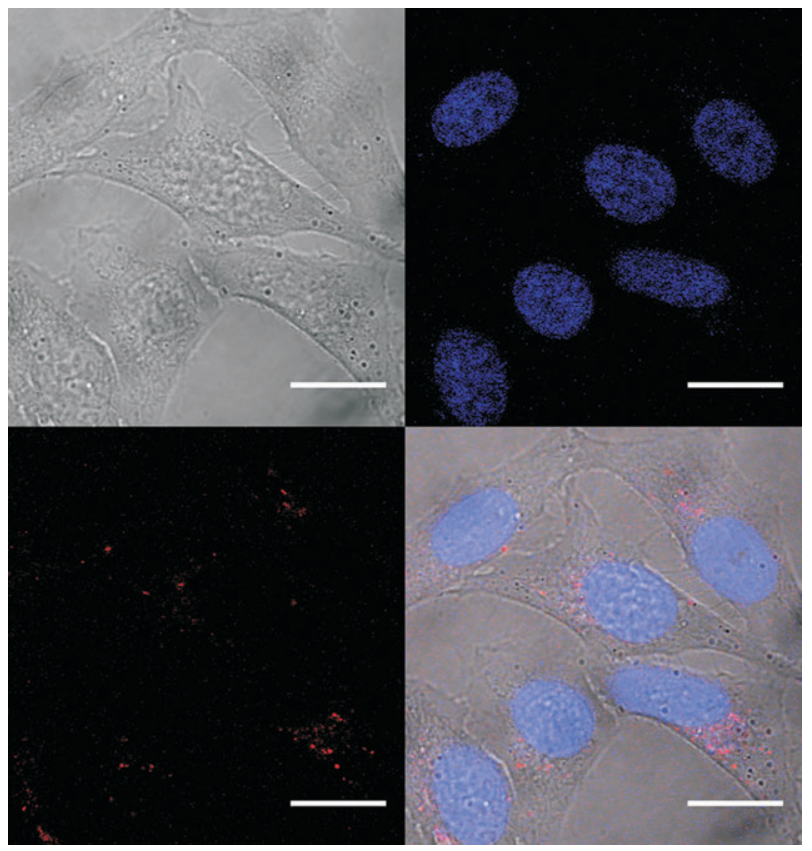

Fig. 2 Images of live HeLa cells labeled with $\mathbf{A r g}_{\mathbf{9}} \mathbf{- 8}$. Upper left: transmission bright-field; upper right: Hoechst 33342 staining of the cell nucleus; bottom left: NIR fluorescence intracellular $\mathbf{A r g}_{9} \mathbf{- 8}$ (excitation wavelength: $633 \mathrm{~nm}$, detection wavelengths: $>650 \mathrm{~nm}$, see ESI $\dagger$ ); bottom right: overlay of Hoechst and $\mathbf{A r g}_{9}-\mathbf{8}$ channels showing the localization of $\mathbf{A r g}_{\mathbf{9}} \mathbf{- 8}$ close to but outside the nucleus, $5 \mathrm{~h}$ after incubation; scale bar: $20 \mu \mathrm{m}$.

(Fig. 2). Additional Hoechst staining shows that these aggregates concentrate around the nucleus, but do not enter inside.

In summary, a synthetic strategy for $\mathrm{BF}_{2}$-PPCys with asymmetric substitution pattern has been described. The compounds show strong NIR absorption and very bright fluorescence. The spectral properties of different derivatives show typical cyanine dye behavior. The asymmetric substitution allows the introduction of one functional group which can be used for labeling applications with NIR excitation and detection. First in vivo experiments show that the resulting dye is brightly fluorescing in live cells and has a very good chemical stability. We did not observe harmful effects on the cells. The findings reported here open exciting possibilities for the derivatization of this new class of compounds and their use in ultrasensitive microscopy in live cells tissues.

Support by the Bioimaging Center of the University of Konstanz and the SFB 767, as well as by C. Strasser for help with the cellular samples, is gratefully acknowledged.

\section{Notes and references}

1 (a) R. Y. Tsien, L. Ernst and A. Waggoner, in Handbook of Biological Confocal Microscopy, ed. J. B. Pawley, Springer, New York, 2006, pp. 338-352; (b) J. Lippincott-Schwartz and G. H. Patterson, Science, 2003, 300, 87.

2 M. S. T. Gonçalves, Chem. Rev., 2009, 109, 190.

3 (a) R. Weissleder, Nat. Biotechnol., 2001, 19, 316; (b) Near-Infrared Dyes for High Technology Applications, NATO Series 3, ed. S. Dähne, U. Resch-Genger, O. S. Wolfbeis, Kluwer, Dordrecht, 1998, vol. 52.

4 (a) A. Becker, C. Hessenius, K. Licha, B. Ebert, U. Sukowski, W. Semmler, B. Wiedenmann and C. Grötzinger, Nat. Biotechnol., 2001, 19, 327; (b) R. Weissleder, C. H. Tung, U. Mahmood and A. Bogdanov, Nat. Biotechnol., 1999, 17, 375; (c) Y. Ye, S. Bloch, B. Xu and S. Achilefu, J. Med. Chem., 2006, 49, 2268.

5 (a) J. O. Escobedo, O. Rusin, S. Lim and R. M. Strongin, Curr. Opin. Chem. Biol., 2010, 14, 64; (b) J. J. Gassensmith, J. M. Baumesm and B. D. Smith, Chem. Commun., 2009, 6329; (c) A. B. Descalzo, H.-J. Xu, Z. Shen and K. Rurack, Ann. N. Y. Acad. Sci., 2008, 1130, 164; (d) M. Taniguchi, D. L. Cramer, A. D. Bhise, H. L. Kee, D. F. Bocian, D. Holten and J. S. Lindsey, New J. Chem., 2008, 32, 947.

6 (a) G. M. Fischer, A. P. Ehlers, A. Zumbusch and E. Daltrozzo, Angew. Chem., Int. Ed., 2007, 46, 3750; (b) G. M. Fischer, M. Isomäki-Krondahl, I. Göttker-Schnetmann, E. Daltrozzo and A. Zumbusch, Chem.-Eur. J., 2009, 15, 4857.

7 M. Y. Berezin, W. J. Akers, K. Guo, G. M. Fischer, E. Daltrozzo, A. Zumbusch and S. Achilefu, Biophys. J., 2009, 97, L22.

8 (a) A. Iqbal, M. Jost, R. Kirchmayer, J. Pfenninger, A. Rochat and O. Wallquist, Bull. Soc. Chim. Belg., 1988, 97, 615; (b) A. C. Rochat, A. Iqbal and O. Wallquist, US Pat., 5,017,706, 1989; (c) F. Closs and R. Gompper, Angew. Chem., Int. Ed., 1987, 26, 552 .

9 (a) L. G. S. Brooker and R. H. Sprague, J. Am. Chem. Soc., 1945, 67, 1869; (b) E. Daltrozzo and W. Sulger, Methine Dyes for Optical Recording Materials, EP0217245B1, Int. Cl. C09B 23/10, G11B7/ 24, Patentbl.87/15, 1992, pp. 1-88; (c) J. Fabian, J. Prak. Chem., 1991, 333, 197; (d) E. Daltrozzo and A. Reiß, New Fluorescence Dyes and Their Use as Fluorescence Marker, US Pat., 6, 552, 199B1, 2003, pp. 1-40; EP 1 054039A1.

10 (a) G. T. Hermanson, Bioconjugate Techniques, Academic Press, 1996; (b) A. El-Faham, R. S. Funosas, R. Prohens and F. Albericio, Chem.-Eur. J., 2009, 15, 9404.

11 P. Lundberg and Ü. Langel, J. Mol. Recognit., 2003, 16, 227.

12 T. Holm, H. Johansson, P. Lundberg, M. Pooga, M. Lindgren and U. Langel, Nat. Protocols, 2006, 1, 1001. 